UM-P-97/49

RCHEP-97/09

\title{
Monte Carlo simulation of systems with complex-valued measures
}

\author{
J.F. Markham and T. D. Kieu \\ School of Physics, University of Melbourne, Vic 3052, Australia
}

A simulation method based on the RG blocking is shown to yield statistical errors smaller than that of the crude MC using absolute values of the original measures. The new method is particularly suitable to apply to the sign problem of indefinite or complex-valued measures. We demonstrate the many advantages of this method in the simulation of $2 \mathrm{D}$ Ising model with complex-valued temperature.

\section{THE SIGN PROBLEM}

In order to evaluate a multi-dimensional integral of the partition function

$Z \equiv \int f d V$

using Monte Carlo (MC) one can sample the points in the integration domain with a nonuniform distribution, $p$. This sampling gives the following estimate for the integral:

$Z \approx\langle f / p\rangle \pm \sqrt{S / N}$

where $N$ is the number of points sampled, $p \geq 0$ and is normalised;

$$
\langle f / p\rangle \equiv \frac{1}{N} \sum_{i=1}^{N} f\left(x_{i}\right) / p\left(x_{i}\right) .
$$

and

$$
\begin{aligned}
S & \equiv \int|f / p-Z|^{2} p d V \\
& \approx\left\langle f^{2} / p^{2}\right\rangle-\langle f / p\rangle^{2} .
\end{aligned}
$$

The best choice of $p$ is the one that minimises the standard deviation squared $S$. This can be found by variational method leading to the crude average-sign $\mathrm{MC}$ weight

$p_{\text {crude }}=|f| / \int|f| d V$,

giving the optimal

$S_{\text {crude }}=\left(\int|f| d V\right)^{2}-\left|\left(\int f d V\right)\right|^{2}$.
The sign problem [1] arises when $Z / \int|f| d V$ is vanishingly small: then unless a huge number of configurations are MC sampled, the large statistical fluctuations of the partition function render the measurement meaningless.

\section{IMPROVED SIMULATION METHOD}

One way of smoothing out the sign problem is to do part of the integral analytically, and the remainder using MC. The analytical summation is not just directly over a subset of the dynamical variables; in general it can be a renormalisation group (RG) blocking where coarse-grained variables are introduced. This does yield certain improvement over the crude MC in general.

Let $P\left\{V^{\prime}, V\right\}$ be the normalised RG weight relating the original variables $V$ to the blocked variables $V^{\prime}$,

$$
\begin{aligned}
P\left\{V^{\prime}, V\right\} & \geq 0, \\
\int P\left\{V^{\prime}, V\right\} d V^{\prime} & =1 .
\end{aligned}
$$

Inserting this unity resolution into the integral (1)

$$
\begin{aligned}
I & =\int d V \int d V^{\prime} P\left\{V^{\prime}, V\right\} f, \\
& \equiv \int d V^{\prime} g\left(V^{\prime}\right) .
\end{aligned}
$$

Thus, an MC estimator is only needed for the remaining integration over $V^{\prime}$ in (6). As with the crude method of the last section, variational 
minimisation for $S$ of (3), with $g$ in place of $f$, leads to the improved MC

$p_{\text {improved }}=|g| / \int|g| d V^{\prime}$.

Firstly, the improved weight sampling yields a partition function of magnitude not less than that sampled by the crude weight:

$$
\begin{aligned}
\mid\langle\langle\text { sign }\rangle\rangle_{\text {improved }} \mid & \equiv Z / \int|g| d V^{\prime} \\
& =\frac{Z}{\int\left|\int P\left\{V^{\prime}, V\right\} f d V\right| d V^{\prime}} \\
& \geq Z / \int|f| d V \\
& \equiv \mid\langle\langle\text { sign }\rangle\rangle_{\text {crude }} \mid
\end{aligned}
$$

Secondly, it is also not difficult to see that the statistical fluctuations associated with improved $\mathrm{MC}$ is not more than that of the crude $\mathrm{MC}$,

$$
\begin{aligned}
& S_{\text {improved }}-S_{\text {crude }} \\
& =\int\left|\frac{g^{2}}{p_{\text {improved }}}\right| d V^{\prime}-\int\left|\frac{f^{2}}{p_{\text {crude }}}\right| d V, \\
& =\left(\int|g| d V^{\prime}\right)^{2}-\left(\int|f| d V\right)^{2}, \\
& \leq 0,
\end{aligned}
$$

Thus the RG blocking always reduces the statistical fluctuations of an observable measurement by reducing the magnitude of $\sqrt{S} / \mid\langle\langle$ sign $\rangle\rangle \mid$.

Note that the special case of equality in (8.9) occurs iff there was no sig $\mathrm{n}$ problem to begin with. How much improvement one can get out of the new MC weight depends on the details of the RG blocking and on the original measure $f$.

\section{COMPLEX 2D ISING MODEL}

The Hamiltonian for the Ising model on a square lattice is

$H=-j \sum_{\langle n n \prime\rangle} s_{n} s_{n \prime}-h \sum_{n} s_{n}$.

Here we allow $j$ and $h$ to take on complex values in general. For the finite lattice, periodic boundary conditions are used.

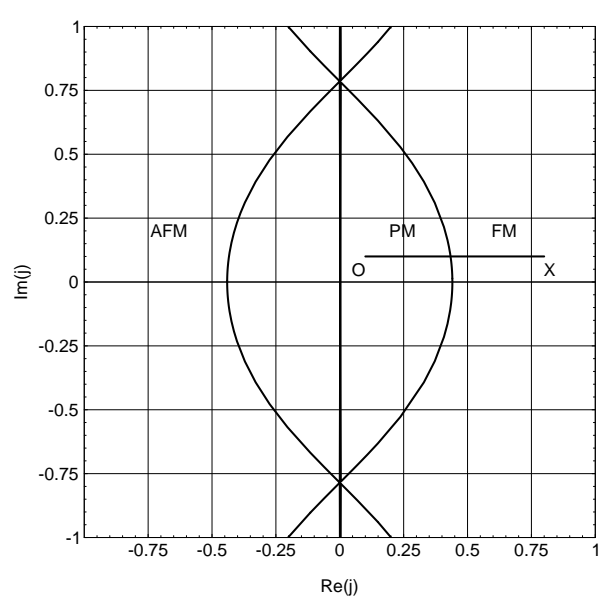

Figure 1. Phase diagram in the complex $j$ plane.

The phase boundaries for the complex temperature 2D Ising model with $h=0$ are depicted in Figure 1 [2].

For the improved method, we adopt a simple RG blocking over the odd sites, labeled $\circ$. That is, the analytic summation is done over the configuration space spanned by the even o sites; while $\mathrm{MC}$ is used to evaluate the sum over the remaining lattice of the odd • sites. In general, with finite-range interactions between the spins, one can always subdivide the lattice into sublattices, on each of which the spins are (nearly) independent for the partial sum to be carried out.

Summing over the spins $s_{\circ}$,

$Z=\sum_{\left\{s_{\bullet}\right\}} e^{h \sum_{\bullet \text { sites }} s_{\bullet}} \prod_{\text {osites }} 2 \cosh \left[j s_{\bullet}^{+}+h\right]$

where $s_{\bullet}^{+}$is the sum of the spins of the four odd neighbours surrounding each even site. The improved MC weight is then the absolute value of the summand on the right hand side of the last expression for $Z$.

The quantities measured are magnetisation, 


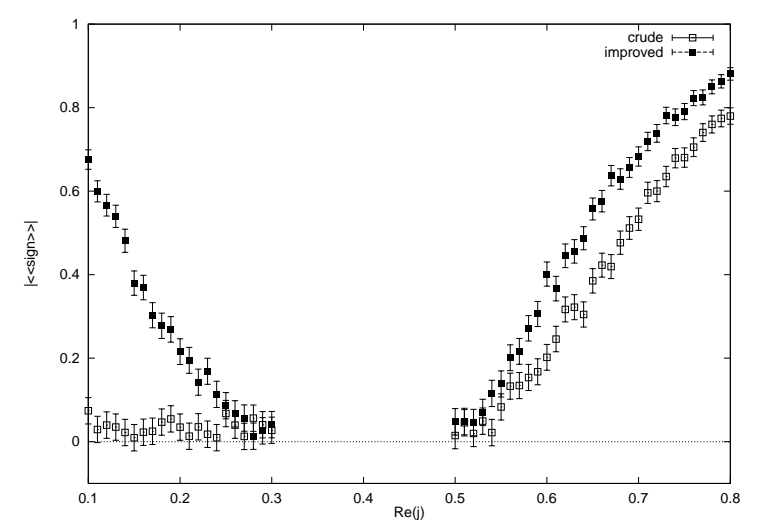

Figure 2. $|<<\operatorname{sign}>>|$ vs $\operatorname{Re}(j) \operatorname{Im}(j)=0$, $h=0$. Filled boxes are the improved data.

$M$, and susceptibility, $\chi$. These can be expressed in terms of the first and second derivatives of $Z$ respectively, evaluated at $h=0$. In all the simulations, square two-dimensional lattices of various sizes with periodic boundary conditions are used. The heat-bath algorithm is used. Two additional benefits arise from the improved method. The first is that the number of sites to be visited is halved. While the expressions to be calculated at the remaining sites turn out to be far more complicated, the use of table look-up means that evaluating them need not be computationally more expensive. The second benefit is that the number of sweeps required to decorrelate data points is reduced.

As a test of the improved method, it is compared to the crude one along the path $O X$ in Figure 1. Comparison with series-expansion data are plotted in Figure 3, in which the discs are the absolute-value circles of complex-valued statistical errors for simulated results.

\section{CONCLUDING REMARKS}

The sign problem is partially eleviated in the improved method [3] because of some partial phase cancellation among the original indefinite

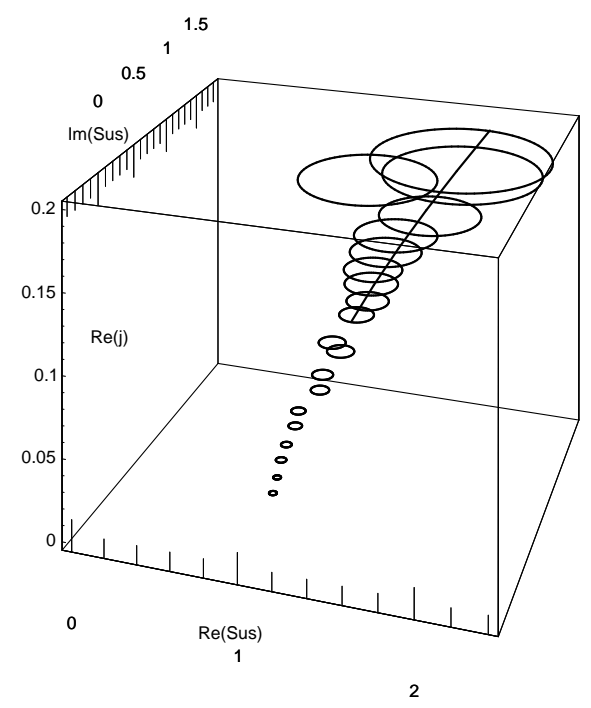

Figure 3. Improved $\chi$ vs $R e(j)$; expansion data shown as line.

or complex-valued measure after an exact RG transformation. A particular RG blocking is chosen for our illustrative example of the 2D Ising model with complex-valued measure. But other choices of RG blocking are feasible and how effective they are depends on the physics of the problems.

We are indebted to Robert Shrock and Andy Rawlinson for help and discussions. We also acknowledge the Australian Research Council and Fulbright Program for financial support.

\section{REFERENCES}

1. For a recent review, see W. von der Linden, Phys. Rep. 220 (1992) 53.

2. V. Matveev and R. Shrock, Phys. Rev. E53 (1996) 254.

3. For the full report, see J.F. Markham and T.D. Kieu, hep-lat/9610006. 